\title{
Characteristics of Macroreentries Using an Epicardial Bypass: Pseudo-Focal Atrial Tachycardia Case Series
}

Yosuke Nakatani ${ }^{1}$, Takashi Nakashima ${ }^{2}$, Josselin Duchateau ${ }^{3}$, Konstantinos Vlachos ${ }^{4}$, Philipp Krisai ${ }^{5}$, Takamitsu Takagi ${ }^{6}$, Tsukasa Kamakura ${ }^{7}$, Clémentine André ${ }^{8}$, Cyril Goujeau $^{9}$, F. Daniel Ramirez ${ }^{10}$, Remi Chauvel ${ }^{11}$, Romain Tixier ${ }^{3}$, Masateru Takigawa ${ }^{12}$, Takeshi Kitamura ${ }^{13}$, Ghassen Cheniti ${ }^{14}$, Arnaud Denis ${ }^{15}$, Frederic Sacher ${ }^{16}$, Mélèze Hocini $^{17}$, Michel Haissaguerre ${ }^{18}$, Pierre Jais $^{3}$, Nicolas Derval ${ }^{19}$, and Thomas Pambrun ${ }^{20}$

${ }^{1}$ University of Toyama

${ }^{2}$ 1. Electrophysiology and Ablation Unit and L'Institut de rythmologie et modélisation cardiaque (LIRYC)

${ }^{3}$ Centre Hospitalier Universitaire de Bordeaux

${ }^{4}$ Evangelismos General Hospital of Athens

${ }^{5}$ University of Bordeaux

${ }^{6}$ Hôpital Cardiologique du Haut- Lévêque, CHU Bordeaux, L'Institut de RYthmologie et modélisation Cardiaque (LIRYC), Université Bordeaux, Bordeaux

${ }^{7}$ National Cerebral and Cardiovascular Center

${ }^{8} \mathrm{CHU}$ Trousseau

${ }^{9}$, Service de Rhythmologie, Hôpital Cardiologique du Haut-Lévêque (Centre Hospitalier Universtaire de Bordeaux)

${ }^{10}$ Centre Hospitalier Universitaire de Bordeaux Hopital Cardiologique

${ }^{11}$ Hôpital Cardiologique du Haut-Lévêque (Centre Hospitalier Universtaire de Bordeaux)

${ }^{12}$ Yokosuka Kyosai Hospital

${ }^{13}$ IHU LIRYC, University of Bordeaux, CHU de Bordeaux

${ }^{14}$ Hôpital Cardiologique du Haut Lévêque

${ }^{15} \mathrm{CHU}$ Bordeaux

${ }^{16}$ Bordeaux University Hospital

${ }^{17}$ Hôpital Cardiologique du Haut-Lévèque

${ }^{18}$ Hopital Cardiologique du Haut-Leveque

${ }^{19}$ Hopital cardiologique du haut-leveque

${ }^{20}$ Hopital du Haut Leveque/LIRYC, Bordeaux

June 15, 2021

\begin{abstract}
Introduction: Human atria comprise distinct epicardial layers, which can bypass endocardial layers and lead to downstream centrifugal propagation at the "epi-endo" connection. We sought to characterize anatomical substrates, electrophysiological properties, and ablation outcomes of "pseudo-focal" atrial tachycardias (ATs), defined as macroreentrant ATs mimicking focal ATs. Methods and Results: We retrospectively analyzed ATs showing centrifugal propagation with post-pacing intervals (PPIs) after entrainment pacing suggestive of a macroreentry. A total of 26 patients had pseudo-focal ATs consisting of 15 perimitral,
\end{abstract}


7 roof-dependent, and 5 cavotricuspid isthmus (CTI)-dependent flutters. A low-voltage area was consistently found at the collision site and co-localized with epicardial layers like the: (1) coronary sinus-great cardiac vein bundle (22\%); (2) vein of Marshall bundle (15\%); (3) Bachmann bundle (22\%); (4) septopulmonary bundle (15\%); (5) fossa ovalis (7\%); and (6) low right atrium (19\%). The mean missing tachycardia cycle length (TCL) was $67 \pm 29 \mathrm{~ms}(22 \%)$ on the endocardial activation map. PPI was 9 [0-15] ms and 10 [0-20] ms longer than TCL at the breakthrough site and the opposite site, respectively. While feasible in 25 pseudo-focal ATs (93\%), termination was better achieved by blocking the anatomical isthmus than ablating the breakthrough site $[24 / 26(92 \%)$ vs. $1 / 6(17 \%)$; p < 0.001]. Conclusion: Perimitral, roof-dependent, and CTI-dependent flutters with centrifugal propagation are favored by a low-voltage area located at well-identified epicardial bundles. Comprehensive entrainment pacing maneuvers are crucial to distinguish pseudo-focal ATs from true focal ATs. Blocking the anatomical isthmus is a better therapeutic option than ablating the breakthrough site.

\section{Characteristics of Macroreentries Using an Epicardial Bypass:}

\section{Pseudo-Focal Atrial Tachycardia Case Series}

Short title: Nakatani, et al. Macroreentries with epicardial bypass

Yosuke Nakatani, MD; Takashi Nakashima, MD; Josselin Duchateau, MD; Konstantinos Vlachos, MD; Philipp Krisai, MD; Takamitsu Takagi, MD; Tsukasa Kamakura, MD; Clémentine André, MD; Cyril Goujeau, MD; F. Daniel Ramirez, MD; Remi Chauvel, MD; Romain Tixier, MD; Masateru Takigawa, MD; Takeshi Kitamura, MD; Ghassen Cheniti, MD; Arnaud Denis, MD; Frédéric Sacher, MD; Mélèze Hocini, MD; Michel Haïssaguerre, MD; Pierre Jaïs, MD; Nicolas Derval, MD; Thomas Pambrun, MD

Hôpital Cardiologique du Haut-Lévêque, CHU Bordeaux, L'Institut de RYthmologie et modélisation Cardiaque (LIRYC), Université Bordeaux, France.

Data availability statement: The data that support the findings of this study are available on request from the corresponding author. The data are not publicly available due to privacy or ethical restrictions.

Funding statement: Dr. Nakatani is supported by the JHRS-EHRA Fellowship. Dr. Krisai is supported by the University of Basel, the Mach-Gaensslen foundation, and the Bangerter-Rhyner foundation. Dr. Ramirez is supported by the Canadian Institutes of Health Research Banting Postdoctoral Fellowship. This work is supported by the Agence Nationale de la Recherche (grant number; IHU LIRYC ANR-10-IAHU-04).

Conflict of interest disclosure: Drs. Haïssaguerre, Hocini, Jaïs, Derval, Sacher and Pambrun have received lecture fees from Biosense Webster. Drs. Derval, Sacher and Jaïs have received lecture fees from Boston Scientific.

Address for correspondence: Yosuke Nakatani, MD

Hôpital Cardiologique du Haut-Lévêque, Avenue de Magellan, 33604 Bordeaux-Pessac, France. Telephone: +33-5-57656542; E-mail:yosuke3gbst@gmail.com

\section{ABSTRACT}

Introduction: Human atria comprise distinct epicardial layers, which can bypass endocardial layers and lead to downstream centrifugal propagation at the "epi-endo" connection. We sought to characterize anatomical substrates, electrophysiological properties, and ablation outcomes of "pseudo-focal" atrial tachycardias (ATs), defined as macroreentrant ATs mimicking focal ATs.

Methods and Results: We retrospectively analyzed ATs showing centrifugal propagation with post-pacing intervals (PPIs) after entrainment pacing suggestive of a macroreentry. A total of 26 patients had pseudo-focal ATs consisting of 15 perimitral, 7 roof-dependent, and 5 cavotricuspid isthmus (CTI)-dependent flutters. A low-voltage area was consistently found at the collision site and co-localized with epicardial layers like the: (1) coronary sinus-great cardiac vein bundle (22\%); (2) vein of Marshall bundle (15\%); (3) Bachmann bundle $(22 \%)$; (4) septopulmonary bundle $(15 \%)$; (5) fossa ovalis $(7 \%)$; and (6) low right atrium $(19 \%)$. The mean missing tachycardia cycle length (TCL) was $67 \pm 29 \mathrm{~ms}(22 \%)$ on the endocardial activation map. PPI was 
9 [0-15] ms and 10 [0-20] ms longer than TCL at the breakthrough site and the opposite site, respectively. While feasible in 25 pseudo-focal ATs (93\%), termination was better achieved by blocking the anatomical isthmus than ablating the breakthrough site [24/26 (92\%) vs. $1 / 6(17 \%) ; \mathrm{p}<0.001]$.

Conclusion: Perimitral, roof-dependent, and CTI-dependent flutters with centrifugal propagation are favored by a low-voltage area located at well-identified epicardial bundles. Comprehensive entrainment pacing maneuvers are crucial to distinguish pseudo-focal ATs from true focal ATs. Blocking the anatomical isthmus is a better therapeutic option than ablating the breakthrough site.

Keywords: Atrial tachycardia; Macroreentry; Epicardial connection; Catheter ablation; Entrainment pacing. INTRODUCTION

The advent of high-density mapping has considerably facilitated the diagnosis and treatment of atrial tachycardia (AT). Macroreentrant circuit and bursting focus are at both ends of the mechanistic spectrum and thus necessitate opposite ablation strategies. Perimitral, roof-dependent, and cavotricuspid isthmus (CTI)dependent flutters can be efficiently treated with a linear lesion blocking an anatomical isthmus. Whereas focal ATs require a patchy lesion targeting the source, which most often corresponds to the earliest electrical activity. At the crossroads has recently emerged a category of AT showing centrifugal propagation, while the underlying mechanism is, in fact, macroreentry. ${ }^{1}$ This comes along with a better understanding of the key role of distinct atrial epicardial structures. ${ }^{2}$ Identifying these "pseudo-focal" ATs is crucial in order to opt for the appropriate ablation strategy.

The present study is a retrospective analysis of pseudo-focal ATs, with specific emphasis on the anatomical substrates, electrophysiological properties, and ablation outcomes.

\section{METHODS}

\section{Patient selection}

To identify pseudo-focal ATs, we retrospectively analyzed consecutive patients who underwent AT ablation using a high-density mapping system in our institution from November 2014 to June 2020. Written informed consent was obtained from all patients before the ablation procedure. The study was approved by our ethics committee on human research.

General principle

Patients were established on oral anticoagulation for at least one month prior to ablation. Unfractionated heparin was administered before transseptal puncture, with a target activated clotting time of 300 to $400 \mathrm{~s}$. All procedures were carried out under conscious sedation. The Rhythmia system (Boston Scientific, Marlborough, MA) or the CARTO-3 HD-coloring system (Biosense Webster, Diamond Bar, CA) was used for the highdensity mapping. Three catheters were inserted via the right femoral vein: (1) a steerable decapolar catheter (Dynamic XT, Boston Scientific) inside the coronary sinus (CS) and the great cardiac vein (GCV); (2) an irrigated tip catheter (IntellaNav, Boston Scientific; or Thermocool SmartTouch, Biosense Webster) for ablation; and (3) a multipolar catheter (Orion, Boston scientific; or Pentaray, Biosense Webster) to build the anatomy and record the electrograms of the left atrium (LA) and right atrium (RA). A steerable long sheath (Agilis, St Jude Medical, St Paul, MN) was used to optimize catheter stability during mapping and ablation. Points were automatically acquired when: (1) the tachycardia cycle length (TCL) was stable ( \pm $10 \mathrm{~ms})$; (2) the time variation of a reference electrogram from the CS catheter was stable ( $\pm 5 \mathrm{~ms}$ ); and (3) the beat-to-beat catheter motion was limited ( $<4 \mathrm{~mm}$ with CARTO-3, and $<1 \mathrm{~mm}$ with Rhythmia). The low-voltage electrogram and noise threshold were defined as an amplitude of $<0.5 \mathrm{mV}$ and $<0.03 \mathrm{mV}$, respectively. Point-by-point radiofrequency ablation was performed using power control mode and normal saline irrigation, with the following settings: (1) 30-50 W during 15-30 s at the endocardial aspect of the atria; and (2) 20-25 W during 15-20 s inside the CS or the GCV.

Pseudo-focal AT analysis 
A pseudo-focal AT was diagnosed in the mapped atrium when all the following criteria were met: (1) centrifugal propagation during high-density mapping; (2) a missing TCL of $>10 \%$; (3) a breakthrough site $>10 \mathrm{~mm}$ remote from a collision site; (4) a post-pacing interval (PPI), after entrainment pacing with pacing cycle length of 10-20 ms shorter than TCL, [?] 30 ms longer than TCL at [?] 3 different sites of the considered atrium, including the breakthrough site and a diametrically opposite site. The latter point made the candidate AT compatible with a macroreentry, defined as a large circuit running along four walls of the mapped atrium. Hence, the three types of macroreentries included in this work were perimitral, roofdependent, or CTI-dependent flutters. Of note, an estimated conduction velocity of the epicardial substrate was calculated by dividing the distance from the breakthrough site to the collision site by the missing TCL.

Several epicardial layers have been described in robust anatomical studies. ${ }^{3-7}$ On this basis, we inferred the bypass of pseudo-focal ATs as follows: (1) $C S-G C V$ bundle if the breakthrough was close to the mitral annulus either at the lateral or posterior vestibule, with a PPI-TCL [?] $30 \mathrm{~ms}$ from the GCV to the CS when entrainment pacing was performed; (2) vein of Marshall (VOM) bundle if the breakthrough was along the LA ridge, with a PPI-TCL [?] $30 \mathrm{~ms}$ at the CS but $>30 \mathrm{~ms}$ at the GCV when entrainment pacing was performed; (3) Bachmann bundle if the breakthrough was at the LA anterior wall or at the anterior edge of the left atrial appendage, and associated with a collision site between the left superior pulmonary vein and the mitral annulus; (4) septopulmonary bundle if the breakthrough was at the LA anterior wall or at the dome between the bilateral pulmonary veins, and associated with a collision site at the roof; (5) fossa ovalis if the breakthrough was at the LA septum close to the transseptal puncture, with PPIs compatible with a perimitral flutter using the RA septum (i.e., bi-atrial flutter); and (6) low RA if the breakthrough was at the inferior portion of the RA lateral wall or septum, and associated with a collision site at the CTI.

Pseudo-focal AT ablation

The ablation target was determined at the discretion of each operator. Two types of strategies were distinguished: (1) obstructing the endocardial emergence with a patchy lesion at the breakthrough site; or (2) blocking an anatomical isthmus of the circuit with a linear lesion, either downstream or upstream of the breakthrough site to avoid or to transect the epicardial bypass. In the latter case, and if the macroreentry was supported by the CS-GCV bundle or the VOM bundle, they were respectively eliminated by endovascular radiofrequency application or by retrograde ethanol infusion as previously described. ${ }^{8,9}$ AT termination was defined as the resumption of sinus rhythm or total alteration of the tachycardia circuit during ablation.

\section{Statistical analysis}

Variables are presented as mean +- standard deviation, median [interquartile range Q1-Q3], or number and percentage, as appropriate. Continuous data were compared using the Student $t$-test or Mann-Whitney U test, depending on data normality. Categorical variables were compared using the $\chi^{2}$ test or Fisher exact test, depending on sample size. Multiple-group comparisons were performed using one-way analysis of variance as a parametric test or Kruskal-Wallis test as a non-parametric test. If significant differences were observed, posthoc tests with Bonferroni-adjusted pairwise comparisons were performed. We considered a $\mathrm{P}$ value $<$ 0.05 as statistically significant.

\section{RESULTS}

Among 655 consecutive patients referred for AT ablation with the high-density mapping system, 26 patients (4\%) exhibited a total of 27 pseudo-focal ATs. Clinical and procedural characteristics are detailed in Table 1 . Briefly, the mean age was $64 \pm 7$ years, 19 patients (73\%) were men, and 24 patients (92\%) had a history of ablation for atrial fibrillation.

Anatomical substrate

The mean distance between the collision site and the breakthrough site was 24 [19-40] mm. In all 27 ATs, the collision site co-localized with a low-voltage area, while the breakthrough site co-localized with an epicardial musculature. The presence of a low-voltage area at the collision site was: (1) spontaneous in 2 patients (8\%) and (2) iatrogenic in 24 patients (92\%). The missing portion of the circuit was bypassed by: (1) the CS-GCV 
bundle (Figure 1 ) in 6 ATs (22\%); (2) the VOM bundle (Figure 2 ) in 4 ATs (15\%); (3) the Bachmann bundle (Figure 3 ) in 6 ATs $(22 \%$ ); (4) the septopulmonary bundle (Figure 4 ) in 4 ATs (15\%); (5) the fossa ovalis (Figure 5 ) in 2 ATs (7\%); and (6) a low RA bridge (Figure 6 ) in 5 ATs (19\%). Details for each type of epicardial bypass are provided in Table 2 .

Electrophysiological properties

A total of 15 perimitral, 7 roof-dependent, and 5 CTI-dependent flutters exhibiting centrifugal propagation were identified by means of high-density mapping associated with conventional entrainment pacing maneuvers. The mean TCL was $303 \pm 65 \mathrm{~ms}$, with a mean missing TCL of $67 \pm 29 \mathrm{~ms}(22 \%)$ on the activation map. The estimated conduction velocity of the epicardial bypass was 49 [29-63] cm/s, and the mean activated area within $10 \mathrm{~ms}$ after the breakthrough was $2.3 \pm 1.5 \mathrm{~cm}^{2}$. Unipolar electrogram recorded at the breakthrough site exhibited an $\mathrm{rS}$ pattern in 17 pseudo-focal ATs (63\%). Details for each type of epicardial bypass are provided in Table 2 .

Ablation outcomes

Ablation of these 27 pseudo-focal ATs required a total of 32 attempts aiming either to target the focal emergence in 6 cases, or to block an anatomical isthmus of the circuit in 26 cases. Termination was achieved in 25 pseudo-focal ATs (93\%). Blocking an anatomical isthmus was significantly more efficient than targeting the endocardial emergence [24/26 (92\%) vs. $1 / 6(17 \%) ; \mathrm{p}<0.001]$. Details for each type of epicardial bypass are shown in Table 2 . After a mean follow-up of $15 \pm 13$ months, arrhythmia recurrence was observed in 9 patients (35\%). During the repeat procedure, the same circuit as in the index procedure was observed in one-third of these patients $(12 \%)$.

\section{DISCUSSION}

The present study retrospectively analyzed the characteristics of pseudo-focal ATs. The main findings are as follows: (1) the breakthrough site is close to a low-voltage area that contains the collision site and masks a well-identified epicardial bundle supporting the concealed part of the circuit; (2) a short PPI at the site opposite to the breakthrough site demonstrates a macroreentrant mechanism despite centrifugal propagation in the activation map; and (3) the distinction from true focal ATs is crucial, since ablating the breakthrough site is not the best therapeutic option in pseudo-focal ATs.

Anatomical substrate: unmasked epicardial bundles

The multilayer architecture of the atria was first described by Papez in $1920 .^{3}$ This key finding was subsequently endorsed by renowned anatomists who gave further insight into the complexity of the atrial musculature. ${ }^{4-7}$ The breakthrough sites observed in the present work were all circumscribed to areas harboring well-identified epicardial bundles or inter-atrial connections. This strongly suggests that pseudo-focal ATs are determined by native anatomical substrates. However, acquired scar substrates may also be crucial for the genesis of pseudo-focal ATs. Hence, we consistently found a low-voltage area constituting a linear obstacle close to the breakthrough site (Figures 1-6 ). This observation confirms previous work by Pathik et al. ${ }^{1}$ Whether due to the aging process or previous ablation, this remodeling is necessary to disrupt the conduction properties of one layer, and thus reveal its electrical dissociation from another layer. Such phenomenon was recently demonstrated with the septoatrial bundle and the septopulmonary bundle, which respectively form the endocardial and the epicardial layers of the dome. The activation of the dome was compared before and after creating a roof line that blocked the endocardial layer only. While initially concealed by their synchronous activation, the electrical dissociation of the two layers was finally unmasked by the sequential activation of the protected septopulmonary bundle first, followed by the septoatrial bundle downstream. ${ }^{10}$ Besides the septopulmonary bundle, other epicardial structures may act similarly, depending on scar location: the CS-GCV and VOM bundles at the mitral isthmus, the Bachmann bundle at the anterior wall, the fossa ovalis at the septum, and the low RA at the CTI.

Electrophysiological diagnosis: key role of entrainment pacing 
Although a minority pattern, a "QS" unipolar electrogram was recorded at the breakthrough site of our series of pseudo-focal ATs, confirming previous observation by Pathik et al. Such pattern is theoretically not compatible with an "epi-endo" breakthrough. However, in clinical practice, unipolar electrograms have been used on the basis of a two-dimensional analysis that considered atrial surface only, without including atrial depth as a third dimension. Thus, the extent to which theory can be applied to the standard recording of a 3-mm thick atrium is unknown, especially if the layers have been previously ablated and are tangentially blending into one another. ${ }^{5}$ This illustrates a limitation of high-density mapping systems if the operator exclusively relies on activation maps based on automatic electrogram recording and annotation.

From this respect, the present study shows that the strongest evidence for a macroreentry is provided by conventional maneuvers as simple as entrainment pacing. Although mostly close to a collision site, our data show that "epi-endo" breakthrough sites can sometimes be more than $50 \mathrm{~mm}$ remote from a collision site. Hence, entrainment pacing maneuvers should be systematically performed in ATs with centrifugal propagation in order to rule out a macroreentry. Barbhaiya et al. have demonstrated that entrainment pacing with a pacing cycle length of $<20 \mathrm{~ms}$ shorter than TCL resulted in an extremely low risk of AT alteration or termination. ${ }^{11}$ Due to slow conduction at the epicardial bypass, the mean TCL was over 300 ms in our case series. This long TCL might have even reduced the risk.

Ablation strategy: targeting anatomical isthmus

The correct diagnosis of pseudo-focal ATs is critical because it conditions the ablation strategy and success. If misdiagnosed as a focal AT, the breakthrough site will be ablated in a macroreentrant AT with centrifugal propagation. Since "epi-endo" connections were usually broad, we observed a poor termination rate (17\%) with a progressive shift of the endocardial emergence as the patchy lesion expands.

From this respect, entrainment pacing maneuvers shift the focus from the breakthrough to the circuit itself and thus clarify the ablation strategy. Instead of a tailored but challenging ablation of the endocardial emergence, a systematic block at a predefined anatomical isthmus seems more sensible. Moreover, a line of block adds the advantage of a clear endpoint. Two key factors may determine the best candidate isthmus: (1) the ability to transect an epicardial bypass by direct elimination; or (2) the ability to avoid an epicardial bypass by upstream or downstream ablation. For perimitral flutters, the CS-GCV and VOM bundles are now readily eliminated by endovascular ablation and retrograde ethanol infusion, making the mitral isthmus an optimal target for the linear block. ${ }^{12,13}$ For roof-dependent flutters, the septopulmonary bundle obstacle can often be avoided at the lower portion of the dome, making the floor line an alternative target when the roof line is not blocked. ${ }^{10}$ For CTI-dependent flutters, ablation inside the CS drainage system has been described in cases of low RA bridges, but creating a line at a more lateral or septal portion of the CTI proved efficient in the majority of cases in our experience. However, these anatomical isthmuses may not always be transected with conventional ablation. ${ }^{14}$ Nayak et al. recently showed that epicardial access could sometimes be necessary. ${ }^{2}$ This approach requires specific training and a dedicated environment with surgical backup.

Study limitations

The present case series has several limitations. First, the sample size is relatively small. This is partly due to our diagnostic criteria of a macroreentry, based on a minimum of three different sites with short PPI. Another reason is the retrospective nature of the work, leading to the exclusion of some cases with incomplete maps. Together, these two points may result in an underestimation of pseudo-focal ATs prevalence. Second, smaller circuits confined to one atrial wall may also involve an epicardial bypass and share some characteristics of our series. However, their diversity implies a less systematic ablation strategy than that we report for a macroreentry. Third, the detailed course of the epicardial bypass was not directly mapped via subxiphoid access. Since the distance from the breakthrough to the collision site is a surrogate for the bypass length, the measured velocity was an estimation only.

\section{CONCLUSION}

Perimitral, roof-dependent, and CTI-dependent flutters can mimic a focal AT with centrifugal propagation. 
These pseudo-focal ATs result from endocardial scarring at distinct anatomical areas, which favors masked conduction via protected epicardial bundles. Blocking the anatomical isthmus of these well-defined flutters is more efficient than ablating the breakthrough site. Thus, comprehensive entrainment pacing maneuvers are crucial for the correct diagnosis of the macroreentrant mechanism and opt for an adequate ablation strategy.

\section{REFERENCES}

1. Pathik B, Lee G, Sacher F, Haïssaguerre M, Jaïs P, Massoullié G, Derval N, Sanders P, Kistler P, Kalman JM. Epicardial-endocardial breakthrough during stable atrial macroreentry: evidence from ultra-highresolution 3-dimensional mapping. Heart Rhythm 2017;14:1200-1207.

2. Nayak HM, Aziz ZA, Kwasnik A, Lee E, Shatz DY, Tenorio R, Besser SA, Beaser AD, Ozcan C, Upadhyay GA, Tung R. Indirect and direct evidence for 3-D activation during left atrial flutter: anatomy of epicardial bridging. JACC Clin Electrophysiol 2020;6:1812-1823.

3. Papez JW. Heart musculature of the atria. Am J Anat 1920;27:255-277.

4. von Lüdinghausen M, Ohmachi N, Boot C. Myocardial coverage of the coronary sinus and related veins. Clin Anat 1992;5:1-15.

5. Ho SY, Sanchez-Quintana D, Cabrera JA, Anderson RH. Anatomy of the left atrium: implications for radiofrequency ablation of atrial fibrillation. J Cardiovasc Electrophysiol 1999;10:1525-1533.

6. Cabrera JA, Ho SY, Climente V, Sánchez-Quintana D. The architecture of the left lateral atrial wall: a particular anatomic region with implications for ablation of atrial fibrillation. Eur Heart J 2008;29:356-362.

7. Cabrera JA, Sánchez-Quintana D, Farré J, Rubio JM, Ho SY. The inferior right atrial isthmus: further architectural insights for current and coming ablation technologies. J Cardiovasc Electrophysiol 2005;16:402408.

8. Valderrábano M, Chen HR, Sidhu J, Rao L, Ling Y, Khoury DS. Retrograde ethanol infusion in the vein of Marshall: regional left atrial ablation, vagal denervation and feasibility in humans. Circ Arrhythm Electrophysiol 2009;2:50-56.

9. Kitamura T, Vlachos K, Denis A, Andre C, Martin R, Pambrun T, Duchateau J, Frontera A, Takigawa M, Thompson N, Cheniti G, Martin CA, Lam A, Bourier F, Sacher F, Hocini M, Haissaguerre M, Jais P, Derval N. Ethanol infusion for Marshall bundle epicardial connections in Marshall bundle-related atrial tachycardias following atrial fibrillation: the accessibility and success rate of ethanol infusion by using a femoral approach. J Cardiovasc Electrophysiol 2019;30:1443-1451.

10. Pambrun T, Duchateau J, Delgove A, Denis A, Constantin M, Ramirez FD, Chauvel R, Tixier R, Welte N, André C, Nakashima T, Nakatani Y, Kamakura T, Takagi T, Krisai P, Cheniti G, Vlachos K, Bourier F, Takigawa M, Kitamura T, Frontera A, Sacher F, Hocini M, Jaïs P, Haïssaguerre M, Walton RD, Derval N. Epicardial course of the septopulmonary bundle: anatomical considerations and clinical implications for roof line completion. Heart Rhythm 2021;18:349-357.

11. Barbhaiya CR, Kumar S, Ng J, Nagashima K, Choi EK, Enriquez A, Chinitz J, Epstein LM, Tedrow UB, John RM, Stevenson WG, Michaud GF. Avoiding tachycardia alteration or termination during attempted entrainment mapping of atrial tachycardia related to atrial fibrillation. Heart Rhythm 2015;12:32-35.

12. Báez-Escudero JL, Morales PF, Dave AS, Sasaridis CM, Kim YH, Okishige K, Valderrábano M. Ethanol infusion in the vein of Marshall facilitates mitral isthmus ablation. Heart Rhythm 2012;9:1207-1215.

13. Nakashima T, Pambrun T, Vlachos K, Goujeau C, André C, Krisai P, Ramirez FD, Kamakura T, Takagi T, Nakatani Y, Kitamura T, Takigawa M, Roux JR, Cheniti G, Tixier R, Chauvel R, Welte N, Duchateau J, Sacher F, Cochet H, Hocini M, Haïssaguerre M, Jaïs P, Derval N. Impact of vein of Marshall ethanol infusion on mitral isthmus block: efficacy and durability. Circ Arrhythm Electrophysiol 2020;13:e008884. 
14. Chaumont C, Saoudi N, Savouré A, Lațcu DG, Anselme F. Electrophysiologic evidence of epicardial connections between low right atrium and remote right atrial region or coronary sinus musculature: relevance for catheter ablation of typical atrial flutter. J Cardiovasc Electrophysiol 2020;31:2344-2351.

\begin{tabular}{|c|c|c|c|c|c|c|}
\hline & & $\begin{array}{l}\text { All ATs }(\mathbf{n}= \\
\text { 27) }\end{array}$ & $\begin{array}{l}\text { Perimitral } \\
\text { flutter }(\mathrm{n}= \\
15)\end{array}$ & $\begin{array}{l}\text { Roof- } \\
\text { dependent } \\
\text { flutter }(\mathrm{n}= \\
7)\end{array}$ & $\begin{array}{l}\text { CTI- } \\
\text { dependent } \\
\text { flutter }(n= \\
5)\end{array}$ & $P$ value \\
\hline Age, years & Age, years & $64 \pm 7$ & $65 \pm 7$ & $63 \pm 7$ & $61 \pm 8$ & 0.726 \\
\hline Male gender & Male gender & $19(73)$ & $10(67)$ & $6(86)$ & $3(75)$ & 0.641 \\
\hline \multirow[t]{5}{*}{$\begin{array}{l}\text { Post AF } \\
\text { ablation }\end{array}$} & $\begin{array}{l}\text { Post AF } \\
\text { ablation }\end{array}$ & $24(92)$ & $15(100)$ & $6(86)$ & $3(75)$ & 0.186 \\
\hline & $\begin{array}{l}\text { Lateral MI } \\
\text { line }\end{array}$ & $11(42)$ & $9(60)$ & $2(29)$ & $0(0)$ & 0.067 \\
\hline & Roof line & $10(38)$ & $8(53)$ & $1(14)$ & $1(25)$ & 0.179 \\
\hline & CTI line & $14(54)$ & $8(53)$ & $3(43)$ & $3(75)$ & 0.588 \\
\hline & Defragmentation & $13(50)$ & $8(53)$ & $3(43)$ & $2(50)$ & 0.901 \\
\hline $\begin{array}{l}\text { Left atrial } \\
\text { volume, } \mathrm{cm}^{3}\end{array}$ & $\begin{array}{l}\text { Left atrial } \\
\text { volume, } \mathrm{cm}^{3}\end{array}$ & $86 \pm 35$ & $90 \pm 33$ & $65 \pm 32$ & $109 \pm 40$ & 0.156 \\
\hline $\begin{array}{l}\text { Procedure } \\
\text { time, min }\end{array}$ & $\begin{array}{l}\text { Procedure } \\
\text { time, min }\end{array}$ & $\begin{array}{l}260 \\
{[210-330]}\end{array}$ & $\begin{array}{l}260 \\
{[194-360]}\end{array}$ & $\begin{array}{l}270 \\
{[221-275]}\end{array}$ & $\begin{array}{l}250 \\
{[210-353]}\end{array}$ & 0.983 \\
\hline $\begin{array}{l}\text { Fluoroscopy } \\
\text { time, min }\end{array}$ & $\begin{array}{l}\text { Fluoroscopy } \\
\text { time, min }\end{array}$ & $64 \pm 36$ & $70 \pm 35$ & $68 \pm 37$ & $36 \pm 33$ & 0.247 \\
\hline $\begin{array}{l}\text { Mapping } \\
\text { points }\end{array}$ & $\begin{array}{l}\text { Mapping } \\
\text { points }\end{array}$ & $\begin{array}{l}15793 \\
{[10237-26462]}\end{array}$ & $\begin{array}{l}14849 \\
{[10711-20282]}\end{array}$ & $\begin{array}{l}28958 \\
{[17275-30762]}\end{array}$ & $\begin{array}{l}2753 \\
{[1219-16678]^{*}}\end{array}$ & 0.015 \\
\hline $\begin{array}{l}\text { Mapped } \\
\text { chamber } \\
\text { volume, } \mathrm{cm}^{3}\end{array}$ & $\begin{array}{l}\text { Mapped } \\
\text { chamber } \\
\text { volume, } \mathrm{cm}^{3}\end{array}$ & $164 \pm 38$ & $162 \pm 40$ & $176 \pm 28$ & $188 \pm 50$ & 0.413 \\
\hline $\begin{array}{l}\text { Total surface } \\
\text { area, } \mathrm{cm}^{2}\end{array}$ & $\begin{array}{l}\text { Total surface } \\
\text { area, } \mathrm{cm}^{2}\end{array}$ & $114 \pm 20$ & $110 \pm 18$ & $117 \pm 19$ & $157 \pm 24^{+}$ & $<0.001$ \\
\hline $\begin{array}{l}\text { Low-voltage } \\
\text { area, } \mathrm{cm}^{2}\end{array}$ & $\begin{array}{l}\text { Low-voltage } \\
\text { area, } \mathrm{cm}^{2}\end{array}$ & $62 \pm 18$ & $65 \pm 16$ & $61 \pm 20$ & $48 \pm 7$ & 0.133 \\
\hline TCL, ms & $\mathrm{TCL}, \mathrm{ms}$ & $303 \pm 65$ & $297 \pm 70$ & $304 \pm 57$ & $319 \pm 69$ & 0.820 \\
\hline $\begin{array}{l}\text { Entrainment } \\
\text { pacing sites }\end{array}$ & $\begin{array}{l}\text { Entrainment } \\
\text { pacing sites }\end{array}$ & $4[3-5]$ & $4[3-4]$ & $4[3-8]$ & $3[3-5]$ & 0.791 \\
\hline $\begin{array}{l}\text { PPI-TCL at } \\
\text { BT site, ms }\end{array}$ & $\begin{array}{l}\text { PPI-TCL at } \\
\text { BT site, ms }\end{array}$ & $9[0-15]$ & $8[0-15]$ & $0[0-14]$ & 10 [10-20] & 0.438 \\
\hline $\begin{array}{l}\text { PPI-TCL at } \\
\text { opposite } \\
\text { site, ms }\end{array}$ & $\begin{array}{l}\text { PPI-TCL at } \\
\text { opposite } \\
\text { site, ms }\end{array}$ & $10[0-20]$ & $5[0-18]$ & $20[6-30]$ & 20 [10-25] & 0.095 \\
\hline
\end{tabular}

Table 1. Clinical and procedural characteristics

Data are mean $\pm \mathrm{SD}$ or number $(\%)$ of patients or median [interquartile range Q1-Q3]. ${ }^{*} \mathrm{P}<0.050$ and ${ }^{+} \mathrm{P}$ $<0.010$ vs. other tachycardias. AF, atrial fibrillation; BT, breakthrough; CTI, cavotricuspid isthmus; MI, mitral isthmus; PPI, post-pacing interval; TCL, tachycardia cycle length. 


\begin{tabular}{|c|c|c|c|c|c|c|c|}
\hline & $\begin{array}{l}\text { All ATs } \\
(\mathrm{n}=27)\end{array}$ & $\begin{array}{l}\text { CS-GCV } \\
(\mathrm{n}=6)\end{array}$ & $\begin{array}{l}\text { VOM }(\mathrm{n} \\
=4)\end{array}$ & $\begin{array}{l}\mathrm{BB}(\mathrm{n}= \\
6)\end{array}$ & $\begin{array}{l}\text { SPB }(n \\
=4)\end{array}$ & $\begin{array}{l}\text { FO }(n= \\
2)\end{array}$ & $\begin{array}{l}\text { Low RA } \\
(\mathrm{n}=5)\end{array}$ \\
\hline $\begin{array}{l}\text { Perimitral } \\
\text { futter }\end{array}$ & $15(55)$ & $6(100)$ & $3(75)$ & $4(67)$ & $0(0)$ & $2(100)$ & $0(0)$ \\
\hline $\begin{array}{l}\text { Roof- } \\
\text { dependent } \\
\text { flutter }\end{array}$ & $7(26)$ & $0(0)$ & $1(25)$ & $2(33)$ & $4(100)$ & $0(0)$ & $0(0)$ \\
\hline $\begin{array}{l}\text { CTI- } \\
\text { dependent } \\
\text { flutter }\end{array}$ & $5(19)$ & $0(0)$ & $0(0)$ & $0(0)$ & $0(0)$ & $0(0)$ & $5(100)$ \\
\hline $\begin{array}{l}\text { Missing } \\
\text { TCL, ms }\end{array}$ & $67 \pm 29$ & $67 \pm 26$ & $83 \pm 33$ & $49 \pm 25$ & $44 \pm 17$ & $112 \pm 13$ & $71 \pm 31$ \\
\hline $\begin{array}{l}\text { Distance BT } \\
\text { to collision, } \\
\mathrm{mm}\end{array}$ & 24 [19-40] & 18 [17-22] & 30 [19-41] & 28 [23-42] & 25 [23-50] & $52[45-59]$ & 20 [19-38] \\
\hline $\begin{array}{l}\text { Epicardial } \\
\text { bypass } \\
\text { velocity, } \\
\mathrm{cm} / \mathrm{s}\end{array}$ & 49 [29-63] & $26[21-49]$ & $44[20-57]$ & 73 [52-123] & 82 [46-112] & $46[44-49]$ & $36[27-57]$ \\
\hline $\begin{array}{l}\text { First } 10 \\
\text { ms } \\
\text { activation } \\
\text { area, } \mathrm{cm}^{2}\end{array}$ & $2.3 \pm 1.5$ & $2.8 \pm 2.0$ & $1.8 \pm 1.0$ & $2.7 \pm 1.2$ & $2.3 \pm 2.0$ & $2.0 \pm 1.6$ & $2.0 \pm 1.3$ \\
\hline $\begin{array}{l}\text { "rS" elec- } \\
\text { trogram at } \\
\text { BT sites }\end{array}$ & $17(63)$ & $4(67)$ & $3(75)$ & $3(50)$ & $3(75)$ & $2(100)$ & $2(40)$ \\
\hline $\begin{array}{l}\mathrm{RF} \\
\text { application } \\
\text { time, min }\end{array}$ & $20[15-45]$ & 16 [11-31] & 29 [17-47] & 25 [14-37] & 42 [21-61] & 12 [8-16] & 41 [20-50] \\
\hline $\begin{array}{l}\text { Pseudo- } \\
\text { focal ATs } \\
\text { termination }\end{array}$ & $25(93)$ & $5(83)$ & $4(100)$ & $6(100)$ & $4(100)$ & $2(100)$ & $4(80)$ \\
\hline $\begin{array}{l}\text { At the } \\
\text { anatomi- } \\
\text { cal } \\
\text { isthmus }\end{array}$ & $24 / 26$ & $5 / 6$ & $3 / 3$ & $6 / 6$ & $4 / 4$ & $2 / 2$ & $4 / 5$ \\
\hline $\begin{array}{l}\text { At the } \\
\text { break- } \\
\text { through } \\
\text { site }\end{array}$ & $1 / 6$ & $0 / 0$ & $1 / 1$ & $0 / 2$ & $0 / 0$ & $0 / 0$ & $0 / 3$ \\
\hline $\begin{array}{l}\text { Arrhythmia } \\
\text { recurrence }\end{array}$ & $9(33)$ & $2(33)$ & $2(50)$ & $1(17)$ & $2(50)$ & $0(0)$ & $2(40)$ \\
\hline
\end{tabular}

Table 2. Anatomical substrate, electrophysiological properties and ablation outcomes

Data are mean $\pm \mathrm{SD}$ or number $(\%)$ of patients or median [interquartile range Q1-Q3]. AT, atrial tachycardia; BB, Bachmann bundle; BT, breakthrough; CS-GCV, coronary sinus and great cardiac vein; CTI, cavotricuspid isthmus; FO, fossa ovalis; RA, right atrium; SPB, septopulmonary bundle; TCL, tachycardia cycle length; VOM, vein of Marshall. 
Figure 1. Pseudo-focal AT (perimitral flutter) using a CS-GCV bundle epicardial bypass

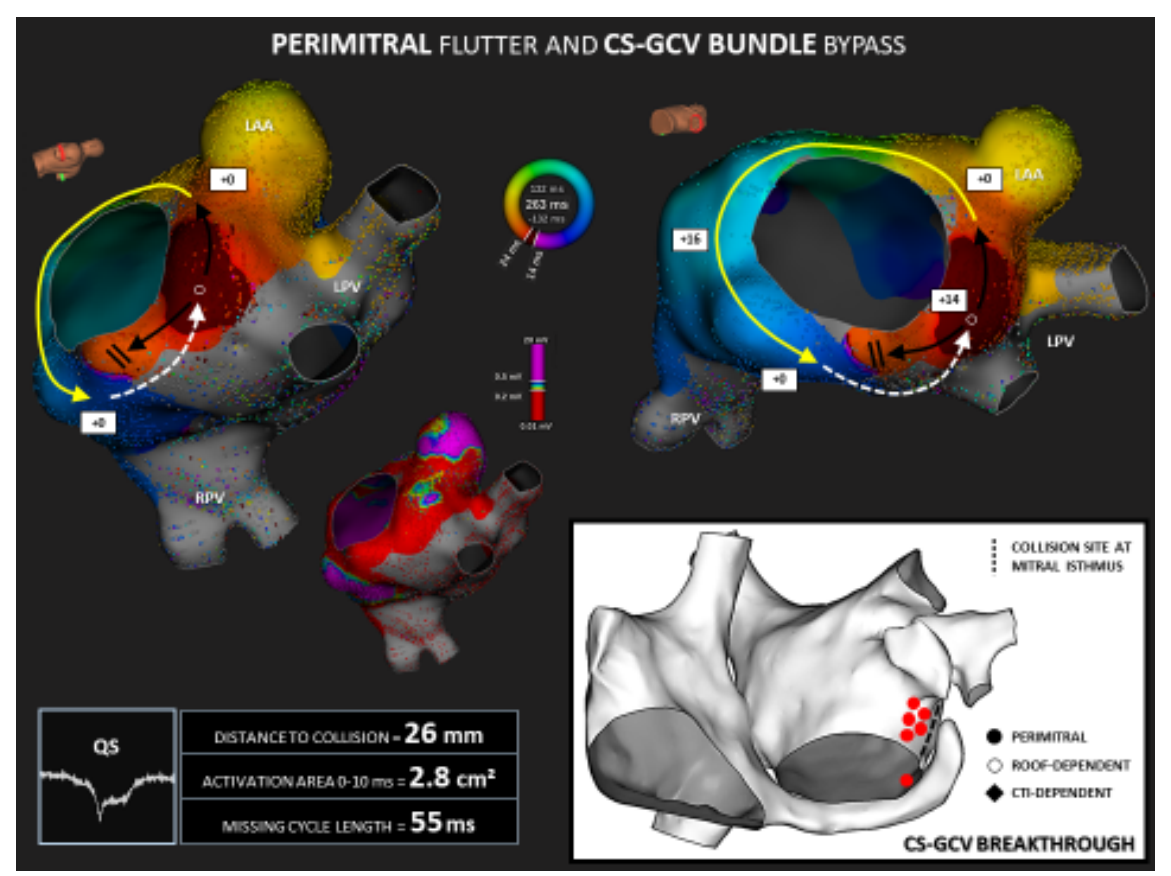

Two different views of the macroreentry activation map. Initial centrifugal propagation (black arrows), subsequent propagation (yellow arrows), and final epicardial bypass (dotted white arrows) over the collision site (double black bars) are displayed. The values of PPI-TCL (white framed numbers ) are shown at each site of entrainment pacing. Associated voltage map $(0.2-0.5 \mathrm{mV})$ and representative unipolar electrogram (white circle) at the breakthrough site are displayed. Distance from breakthrough to collision site, area after $10 \mathrm{~ms}$ of centrifugal activation, and missing cycle length are expressed. Lower right panel : location of the breakthrough sites and type of macroreentry (disc for perimitral,circle for roof-dependent, and losange for CTI-dependent) using this bypass. CTI, cavotricuspid isthmus; LAA, left atrial appendage; LPV, left pulmonary veins; PPI, post-pacing interval; RPV, right pulmonary veins; TCL, tachycardia cycle length.

Figure 2. Pseudo-focal AT (perimitral flutter) using a VOM bundle epicardial bypass 


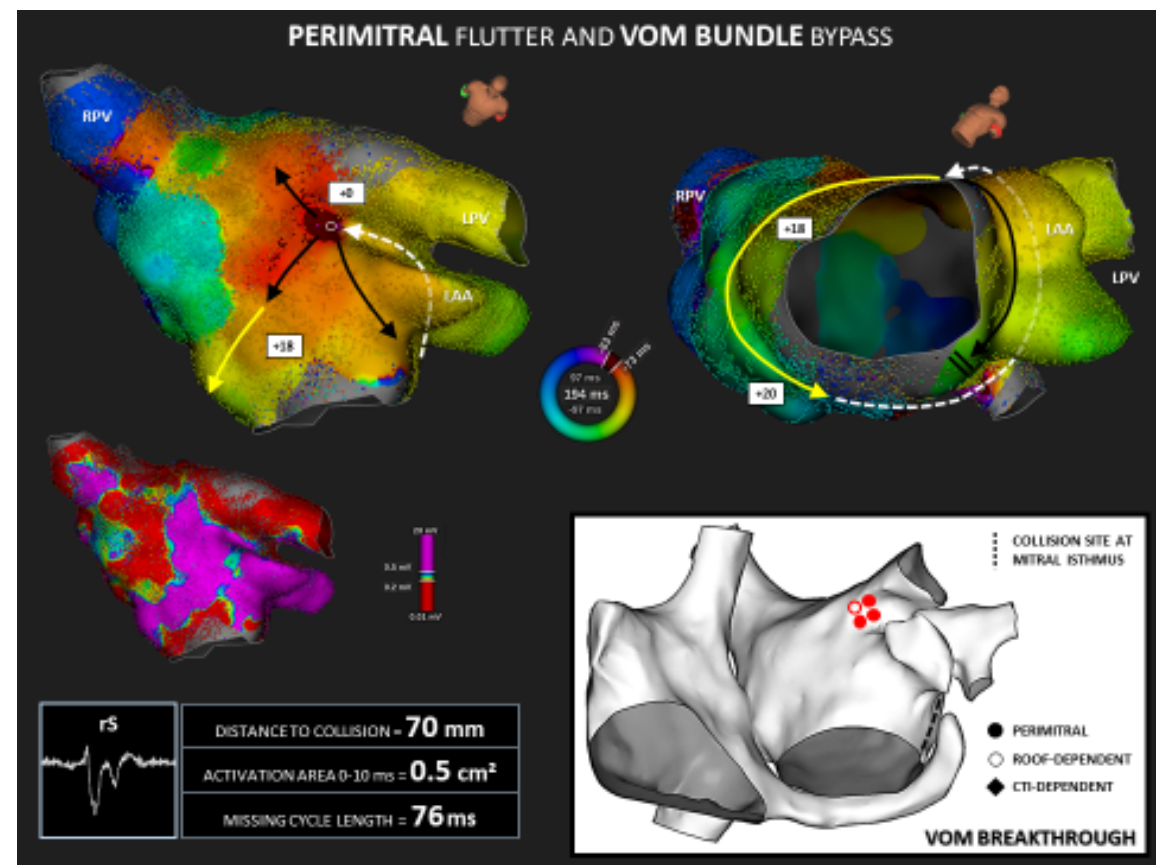

Same legend as Figure 1.

Figure 3. Pseudo-focal AT (perimitral flutter) using a Bachmann bundle epicardial bypass

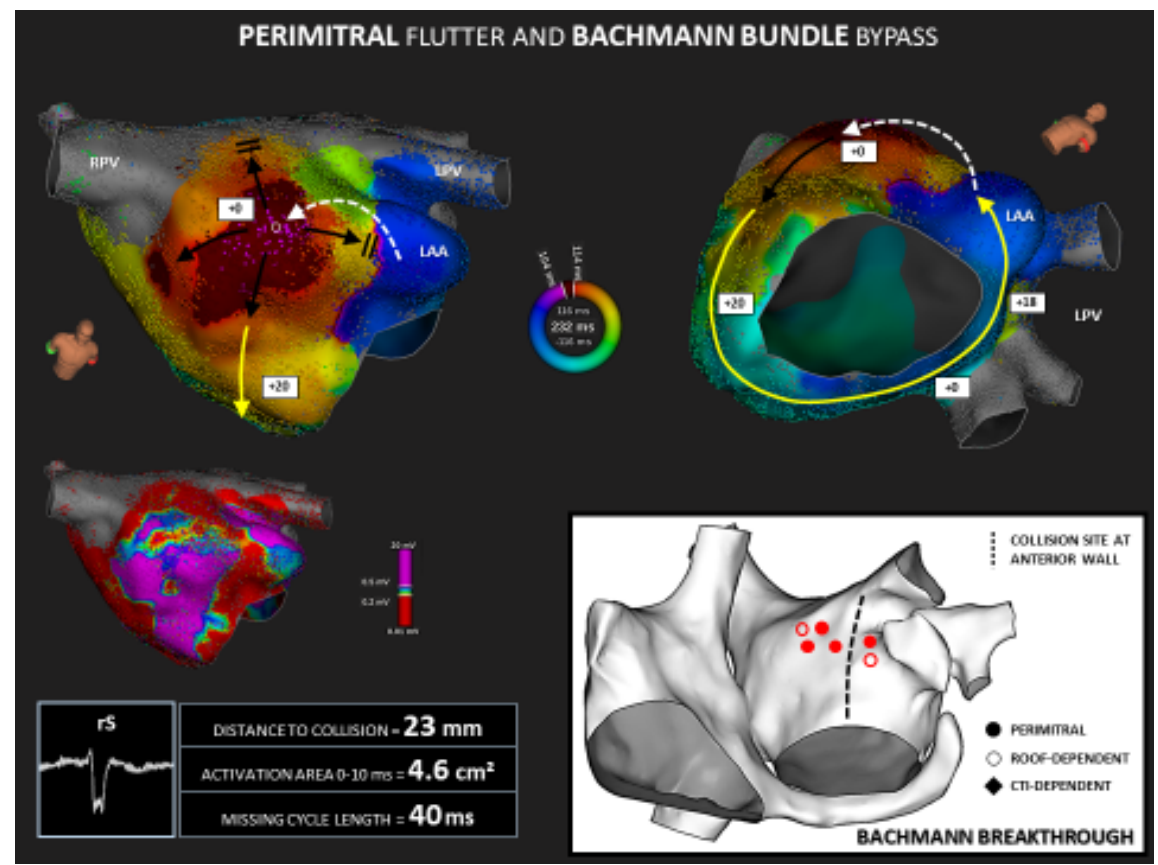

Same legend as Figure 1.

Figure 4. Pseudo-focal AT (roof-dependent flutter) using a septopulmonary bundle bypass 


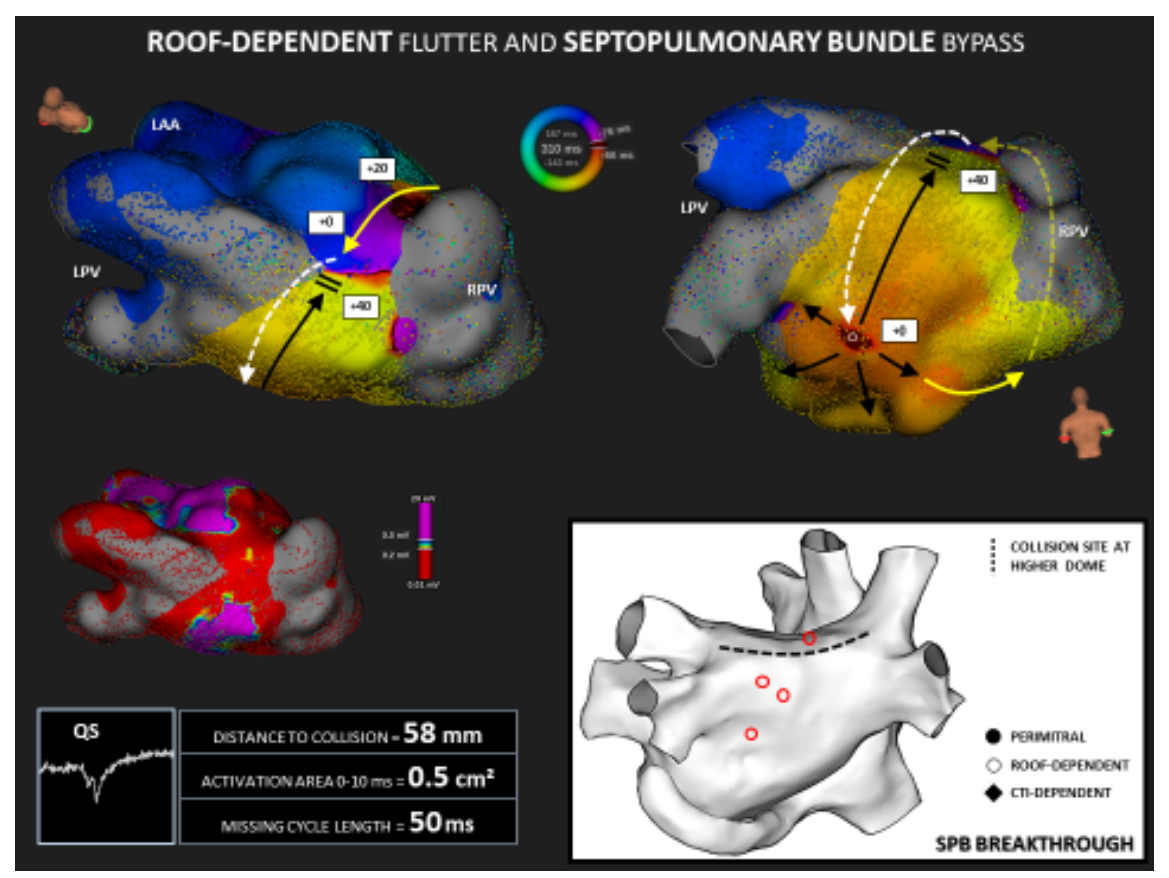

Same legend as Figure 1.

Figure 5. Pseudo-focal AT (perimitral flutter) using a fossa ovalis bypass

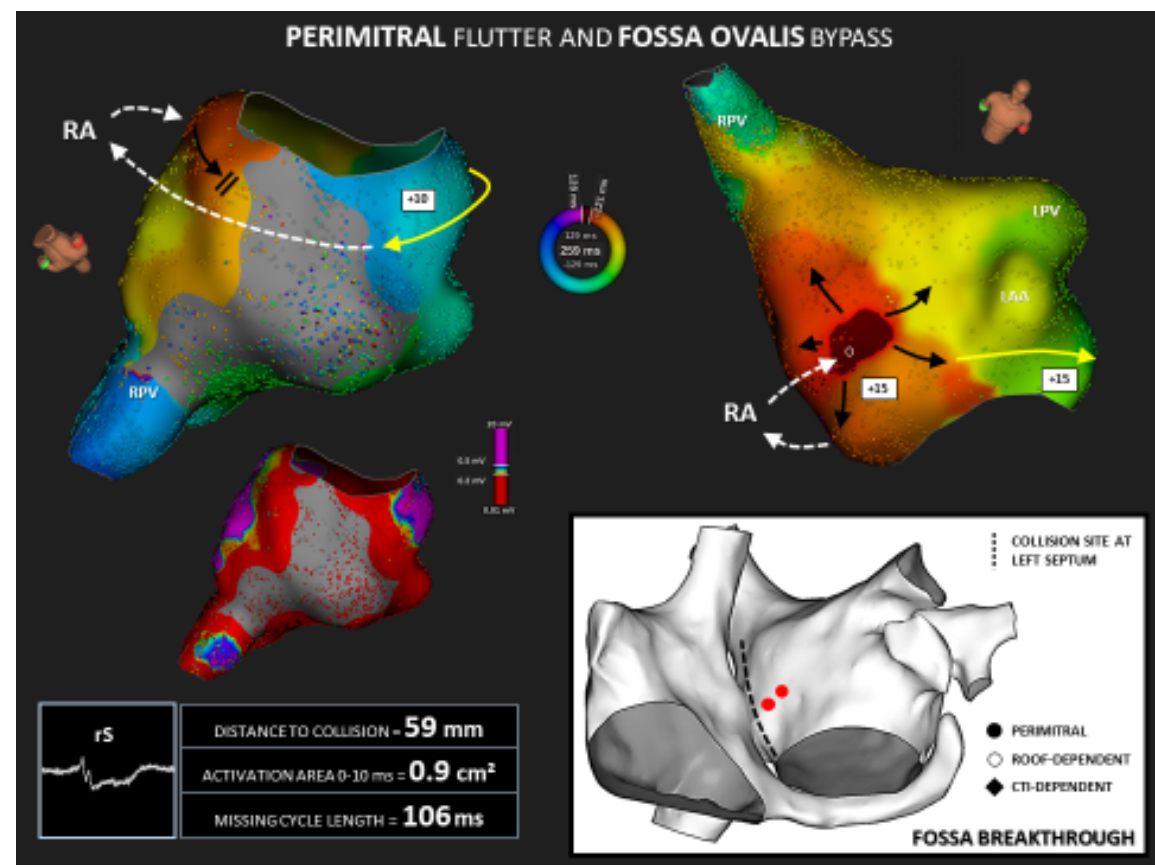

Same legend as Figure 1.

Figure 6. Pseudo-focal AT (CTI-dependent flutter) using a low RA bypass 


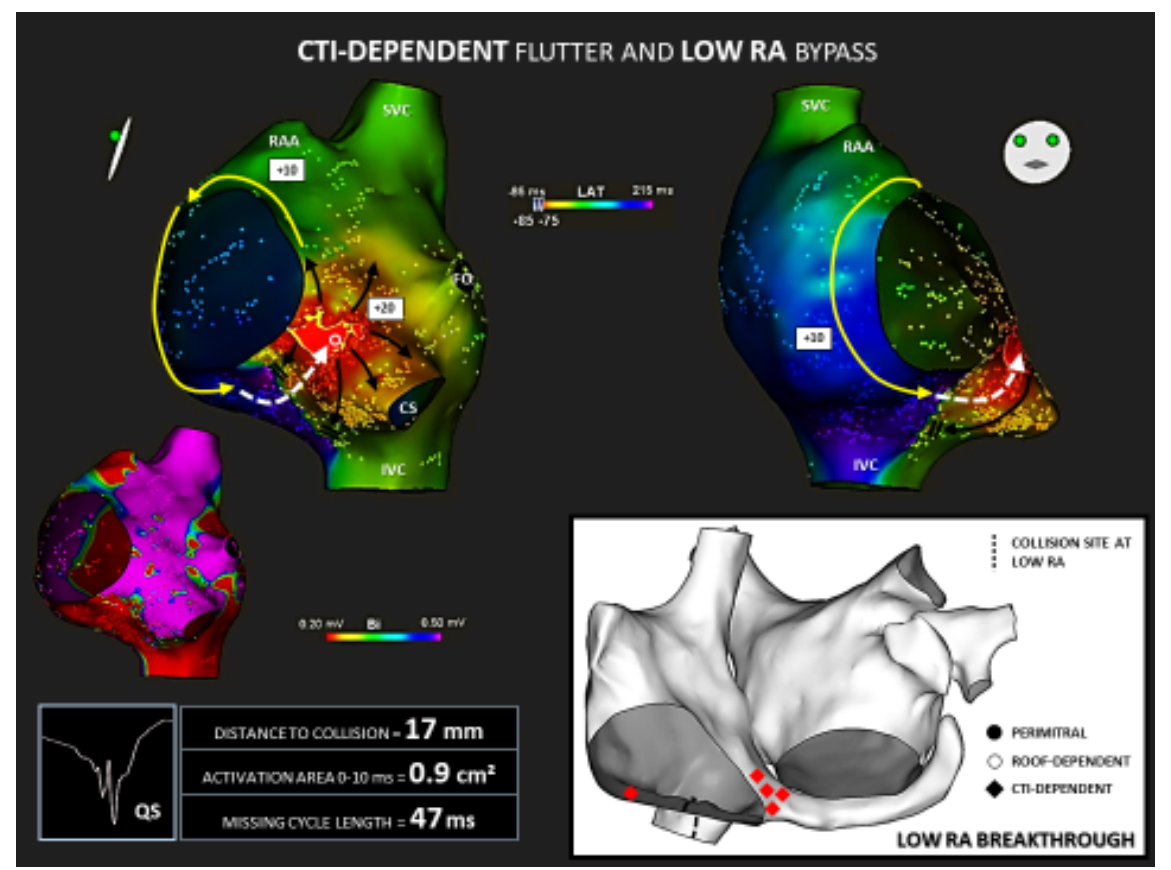

Same legend as Figure 1. CS, coronary sinus; FO, fossa ovalis; IVC, inferior vena cava; RAA, right atrial appendage; SVC, superior vena cava. 